\title{
SHADOWING PROPERTY OF CONTINUOUS MAPS
}

\author{
TOMÁŠ GEDEON AND MILAN KUCHTA
}

(Communicated by Andrew M. Bruckner)

\begin{abstract}
We study continuous maps of an interval into itself. We find the necessary and sufficient condition for the maps of the type $2^{n}$ to have the shadowing property. Further we show that any chaotic map, which has only cycles of order a power of 2 , does not have the shadowing property.
\end{abstract}

\section{INTRODUCTION}

Let $f: I(=\langle 0 ; 1\rangle) \rightarrow I$ be a continuous map of the interval $I$ to itself (i.e., $\left.f \in C^{0}(I, I)\right)$. The orbit of $x$ is the set $\left\{x, f(x), f^{2}(x), \ldots\right\}$ and let $f_{\delta}(x):=\langle f(x)-\delta ; f(x)+\delta\rangle$. Given $\delta>0$, a $\delta$-chain of $x$ (or $\delta$-pseudo orbit of $x$ ) is a sequence $\mathbf{X}_{\delta}=\left\{\mathbf{x}_{n}\right\}_{n=0}^{\infty}$, where $\mathbf{x}_{i+1} \in f_{\delta}\left(\mathbf{x}_{i}\right)$ and $\mathbf{x}_{0}=x$.

The investigation of the pseudo orbits is very important in connection with the calculation of the orbits by a computer, because a computer can calculate only pseudo orbits. For example, if a computer has accuracy in computation of $f(x)$ to 20 decimal places, we can regard $\mathbf{x}_{i+1}$ as the truncation of $f\left(\mathbf{x}_{i}\right)$ to 20 decimal places so that $\left|\mathbf{x}_{i+1}-f\left(\mathbf{x}_{i}\right)\right|<2^{-20}$. Therefore it is very interesting to know when the pseudo orbits can be approximated by actual orbits.

We say that $\delta$-chain $\mathbf{X}_{\delta}$ is $\varepsilon$-shadowed by an orbit of $y$ if for every $n$

$$
\left|\mathbf{x}_{n}-f^{n}(y)\right|<\varepsilon .
$$

Now, if for every $\varepsilon>0$ there is such a $\delta>0$ that every $\mathbf{X}_{\delta}$ is $\varepsilon$-shadowed by some $y \in I$, then $f$ has the shadowing property.

The main aim of this paper is to find the class of continuous maps with the shadowing property. We will assume that $\operatorname{Per}(f)=\operatorname{Fix}(f)$ and that $\operatorname{Fix}(f)$ is nowhere dense in $I$. While the first assumption is technical and we can easily extend our result to the maps with $\operatorname{Per}\left(f^{n}\right)=\operatorname{Fix}\left(f^{n}\right)$, i.e., on maps of the type $2^{n}$ (see Theorem 2), the second assumption is a necessary one for the shadowing property. We abandoned the very restrictive assumption, that $f$ is a homeomorphism, which was used in [3].

In the paper in [1] the family of tent maps was investigated and the residual

Received by the editors October 3, 1990.

1980 Mathematics Subject Classification (1985 Revision). Primary 54H20, 26A18.

Key words and phrases. Iteration, shadowing property, topological dynamics. 
set of parameters $s$ was found, for which $f_{s}$ has the shadowing property.

\section{PRELIMINARIES}

We will denote a closed interval with $x \leq y$ by $\langle x ; y\rangle$ and a closed interval where no information about order of $x, y$ is provided, by $\langle x ; y\rangle^{*}$.

In the sequel, we will use the following lemmas of Šarkovskii [4]:

Lemma 1 (Šarkovskii [4]). Let $f \in C^{0}(I ; I)$ and $\operatorname{Per}(f)=\operatorname{Fix}(f)$. Then for any $x \in I$, if $f(x)>x(f(x)<x)$, then for all $y \in\langle x ; f(x)\rangle$ (respectively $y \in\langle f(x) ; x\rangle$ ) we have $f(y)>x$ (respectively $f(y)<x$ ).

Lemma 2 (Šarkovskiî [4]). Let $f \in C^{0}(I ; I)$ and $\operatorname{Per}(f)=\operatorname{Fix}(f)$. Then for all $n_{1}, n_{2}, n_{3} \in N ; n_{1}<n_{2}<n_{3}$, and for all $x \in I$, if $f^{n_{2}}(x) \neq f^{n_{3}}(x)$, then $f^{n_{1}}(x) \notin\left\langle f^{n_{2}}(x) ; f^{n_{3}}(x)\right\rangle^{*}$.

Lemma 3 (Šarkovskii [4]). Let $f \in C^{0}(I ; I)$ and $\operatorname{Per}(f)=\operatorname{Fix}(f)$. Then for any $x \in I, \lim _{n \rightarrow \infty} f^{n}(x)=p$, where $p \in \operatorname{Fix}(f)$.

Before stating the main result we prove several lemmas.

Lemma 4. Let $f \in C^{0}(I ; I)$ and $\operatorname{Per}(f)=\operatorname{Fix}(f)$. Then for any $\varepsilon>0$ there exists $\delta>0$ such that for any $x_{1}, x_{2}, x_{3} \in I$ with $x_{1} \in\left\langle x_{3} ; x_{2}\right\rangle^{*}$, and $x_{2} \in$ $f_{\delta}\left(x_{1}\right)$, and $x_{3} \in f_{\delta}\left(x_{2}\right)$, we have $\left|x_{2}-x_{3}\right|_{\bullet}<\varepsilon$. (It is an analogue of Lemma 2.)

Proof. Let

$$
\begin{gathered}
\varepsilon_{\delta}^{1}=\sup \{|f(x)-f(y)| ;|x-y| \leq \delta\} \\
\varepsilon_{\delta}^{2}=\sup \left\{|x-y| ; x \in f_{\delta}(y) \text { and } y \in f_{\delta}(x)\right\} \\
\text { Clearly, if } \delta \rightarrow 0 \text { then } \varepsilon_{\delta}^{1}, \varepsilon_{\delta}^{2} \rightarrow 0 .
\end{gathered}
$$

Without loss of generality assume that $x_{3} \leq x_{1} \leq x_{2}$. We will discuss several cases.

(A) Let $f\left(x_{1}\right) \leq x_{1}$. Then $\mid x_{2}-x_{1} \leq \delta$ and by (1) we have that $\mid f\left(x_{2}\right)-$ $f\left(x_{1}\right) \mid \leq \varepsilon_{\delta}^{1}$. Hence $\left|x_{3}-x_{2}\right| \leq \varepsilon_{\delta}^{1}+2 \delta$.

(B1) Let $f\left(x_{1}\right)>x_{1}$ and $f\left(x_{2}\right) \geq x_{1}$. Since $x_{3} \leq x_{1}$ then $x_{1} \in f_{\delta}\left(x_{2}\right)$ and by (2) we have $\left|x_{1}-x_{2}\right| \leq \varepsilon_{\delta}^{2}$. Finally since $f\left(x_{2}\right) \geq x_{1} \geq x_{3}$ we have $\left|x_{3}-x_{2}\right| \leq \varepsilon_{\delta}^{2}+\delta$.

(B2) Let $f\left(x_{1}\right)>x_{1}$ and $f\left(x_{2}\right)<x_{1}$. From Lemma 1 we have that $x_{2}>$ $f\left(x_{1}\right)$ and so there exists $y \in\left\langle x_{1} ; x_{2}\right\rangle$ such that $f(y)=x_{1}$. Again using Lemma 1 we have $y \in\left\langle f\left(x_{1}\right) ; x_{2}\right\rangle$, then $y \in f_{\delta}\left(x_{1}\right)$ and (2) implies $\left|x_{1}-y\right| \leq \varepsilon_{\delta}^{2}$. Hence $\left|x_{1}-x_{2}\right| \leq \varepsilon_{\delta}^{2}+\delta$.

Finally, since $\left|y-x_{2}\right| \leq \delta$ by (1) we have $\left|x_{1}-f\left(x_{2}\right)\right| \leq \varepsilon_{\delta}^{1}$ and $\left|x_{1}-x_{3}\right| \leq$ $\varepsilon_{\delta}^{1}+\delta$.

Now clearly $\left|x_{2}-x_{3}\right| \leq \varepsilon_{\delta}^{1}+\varepsilon_{\delta}^{2}+2 \delta$. Hence for $x_{1}, x_{2}, x_{3}$ satisfying assumptions of our lemma we have that $\left|x_{2}-x_{3}\right| \leq \varepsilon_{\delta}^{1}+\varepsilon_{\delta}^{2}+2 \delta$ and (3) finishes the proof.

Lemma 5. Let $f \in C^{0}(I ; I)$ and $\operatorname{Per}(f)=\operatorname{Fix}(f)$. Let $0=d_{0}<d_{1}<\cdots<$ $d_{n}=1$ be a division of $I$ such that $d_{i} \notin \operatorname{Fix}(f)$ for $i \in\{1, \ldots n-1\}$. Denote by $I_{j}=\left\langle d_{j-1} ; d_{j}\right\rangle$ for $j \in\{1, \ldots, n\}$. Then there exists such a $\delta>0$ 
that if for some $m \in N$ there are points $x_{1}, \ldots, x_{m}$ with $x_{i+1} \in f_{\delta}\left(x_{i}\right)$ for $i \in\{1, \ldots, m-1\}$ and $x_{1} \in f_{\delta}\left(x_{m}\right)$ (i.e., $x_{1}, \ldots, x_{m}$ are $\delta$-m-cycle), then there is a $j$ such that $x_{i} \in I_{j}$ for every $i$ (i.e., all $\delta$-cycles for sufficiently small $\delta$ are "inside" the intervals $\left.I_{j}\right)$.

Proof. Let $2 \varepsilon=\min \left\{\left|d_{i}-p\right| ; p \in \operatorname{Fix}(f)\right.$ and $\left.i \in\{1, \ldots, n-1\}\right\}$. (Fix $(f)$ is closed set and $d_{i} \notin \operatorname{Fix}(f)$.) For this $\varepsilon$ we can find a $\delta>0$ for which Lemma 4 is satisfied and

$$
x \notin f_{\delta}(x) \text { for every } x \in\left\langle d_{i}-\varepsilon ; d_{i}+\varepsilon\right\rangle
$$

(because $f$ is uniformly continuous).

We claim that this $\delta$ satisfies Lemma 5 . To the contrary suppose that there exists an $\delta$-cycle $x_{1}, \ldots, x_{m}$ and $d:=d_{i}$ such that $d \in\left(x_{j} ; x_{k}\right)^{*}$ for some $j$ and $k$.

Without loss of generality assume that $f(d)>d, x_{1} \leq d$, and $x_{2}>d$ and $x_{1}$ is maximal point with this property, i.e.

$$
\text { if } x_{i} \leq d \text { and } x_{i+1}>d \text { then } x_{i} \leq x_{1} .
$$

Let $x_{j} \geq d, x_{j+1}<d$, and let $j$ be minimal index with this property, i.e.

$$
x_{i} \geq d \text { for } i \in\{2, \ldots, j\} .
$$

Clearly $x_{j}>d+\varepsilon$. We will again discuss two cases.

(A) Suppose $x_{j+1} \leq x_{1}$. Then there exists $k \in\{1, \ldots, j-1\}$ such that $x_{1} \leq x_{k}<x_{j} \leq x_{k+1}\left(\right.$ see (6)) and there is a $y \in\left\langle x_{k} ; x_{j}\right\rangle$ such that $x_{j} \in$ $f_{\delta}(y)$ (it follows from the continuity of the relation $f_{\delta}$ ). So we have $y \in\left\langle x_{j+1} ; x_{j}\right\rangle, x_{j} \in f_{\delta}(y)$, and $\left|x_{j}-x_{j+1}\right|>\varepsilon$, hence a contradiction with Lemma 4.

(B) Now suppose $x_{j+1}>x_{1}$. Then we have $x_{j+1}<d-\varepsilon$ (see (4) and (5)) and then also $x_{1}<d-\varepsilon$. Now from (5) similarly as in (A) we obtain that there is a $y \in\left\langle x_{1} ; d-\varepsilon\right\rangle$ with $x_{1} \in f_{\delta}(y)$. So we have $y \in\left\langle x_{1} ; x_{2}\right\rangle, x_{1} \in f_{\delta}(y)$, and $\left|x_{2}-x_{1}\right|>\varepsilon$-again a contradiction with Lemma 4.

Definition 1. Let us consider $\mathbf{X}_{\delta}=\left\{\mathbf{x}_{i}\right\}_{i=0}^{\infty}$ and define numbers $K^{k}=\sum_{i=0}^{\infty} \alpha_{i k}$ where

$\alpha_{i k}=1$ if there is such $j \in\{0, \ldots, i\}$ that $\mathbf{x}_{i-j} \in \operatorname{int}\left(I_{k}\right), \mathbf{x}_{i+1} \notin I_{k}$, and $\mathbf{x}_{i-j+1} \in I_{k}$ for $1 \leq l \leq j$

$\alpha_{i k}=0$ otherwise

(i.e., $K^{k}$ is the number of departures from $I_{k}$ ).

We will call $K=\sum_{k=1}^{n} K^{k}$ the interval index of $\mathbf{X}_{\delta}$.

Lemma 6. Let $f \in C^{0}(I ; I)$ and $\operatorname{Per}(f)=\operatorname{Fix}(f)$. Let us consider the same division $\left\{d_{i}\right\}_{i=0}^{n}$ as in Lemma 5. Then there exists $\delta>0$ and $N_{0} \in N$ such that the interval index $K$ of every $\mathbf{X}_{\delta}$ is less than $N_{0}$.

Proof. Take a $\delta$ such that all $\delta$-cycles are "inside" the intervals $I_{j}$ (see Lemma $5)$. Assume to the contrary that our statement is not true. Then there exists such an $\mathbf{X}_{\frac{\delta}{2}}$ for which $K>n m$ where $n$ is the number of intervals $I_{j}$ and $m>\max _{j \in\{1, \ldots, n\}}\left\{2\left|I_{j}\right| / \delta\right\}$. 
Then there exists an interval $I_{\alpha}$ for which $K^{\alpha}>m$. Indeed, let $I_{\alpha}$ be an interval for which $K^{\alpha}=\max _{j \in\{1, \ldots, n\}}\left\{K^{j}\right\}$. We have $K^{\alpha} \geq K / n>m$.

If for some $i$ there is such $j \in\{0, \ldots, i\}$ that $\mathbf{x}_{i-j} \in \operatorname{int}\left(I_{\alpha}\right), \mathbf{x}_{i+1} \notin I_{\alpha}$, and $\mathbf{x}_{i-j+l} \in I_{\alpha}$ for $1 \leq l \leq j$, then we will denote such an $\mathbf{x}_{i}$ as $\hat{\mathbf{x}}_{i}$.

But because $K^{\alpha}>m$ then we obtain that there are more than $m$ such a points $\hat{\mathbf{x}}_{i}$ and by the Dirichlet principle we can find such $\hat{\mathbf{x}}_{i}, \hat{\mathbf{x}}_{j} \in I^{\alpha} ; i<j$, that $\left|\hat{\mathbf{x}}_{i}-\hat{\mathbf{x}}_{j}\right|<\delta / 2$ and so $\left|f\left(\mathbf{x}_{j-1}\right)-\hat{\mathbf{x}}_{i}\right| \leq \delta$. Hence $\left\{\hat{\mathbf{x}}_{i}, \mathbf{x}_{i+1}, \ldots, \mathbf{x}_{j-2}, \mathbf{x}_{j-1}\right\}$ is a $\delta$-cycle, which is not in a single interval $I^{\alpha}$. Hence we have a contradiction to our assumption that all $\delta$-cycles are "inside" the intervals $I_{j}$.

Definition 2. We will call a one-side neighborhood $\langle p ; q\rangle^{*}$ of the fixed point $p$ a nontrapping neighborhood of $p$ if for every $x \in\langle p ; q\rangle^{*}$ we have $x \in$ $f\left(\langle p ; x\rangle^{*}\right)$.

Lemma 7. Let $f \in C^{0}(I ; I)$ and $\operatorname{Per}(f)=\operatorname{Fix}(f)$. Let $x \in I$ be such that $f^{n}(x) \rightarrow p, p \in \operatorname{Fix}(f)$, and let $\langle p ; q\rangle^{*}$ be a nontrapping neighborhood of $p$. Then if $f^{n}(x) \in\langle p ; q\rangle^{*}$ for some $n \in N$, then we have $f^{n}(x)=p$.

Proof. Let $f^{n}(x) \in\langle p ; q\rangle^{*}$. Because $\langle p ; q\rangle^{*}$ is a nontrapping neighborhood of $p$ there is a $z \in\left\langle p ; f^{n}(x)\right\rangle^{*}$ such that $f(z)=f^{n}(x)$. But because $f^{i}(x) \rightarrow p$ we obtain from Lemma 2 that $z=p$. Hence $f^{n}(x)=p$.

\section{MAIN RESUlTS}

The following lemma is very technical and is needed in the proof of the Main Theorem.

Lemma 8. Let $f \in C^{0}(I ; I)$ and $\operatorname{Per}(f)=\operatorname{Fix}(f)$. Let $0=d_{0}<d_{1}<\cdots<$ $d_{n}=1$ be the division of $I$ as in Lemma 5. Let us consider some sequence of $\delta$-chains $\left\{\mathbf{X}_{\delta_{j}}\right\}_{j=0}^{\infty}=\left\{\left\{\mathbf{x}_{i}^{j}\right\}_{i=0}^{\infty}\right\}_{j=0}^{\infty}$ where $\lim _{j \rightarrow \infty} \delta_{j}=0$ and $\lim _{j \rightarrow \infty} \mathbf{x}_{0}^{j}=x$.

Let $\lim _{n \rightarrow \infty} f^{n}(x)=p, p \in I_{k}$, and let there exist such $n_{0} \in N$ such that if $n \geq n_{0}$, then $f^{n}(x) \in \operatorname{int}\left(I_{k}\right)$.

Suppose that there is such a $j_{0} \in N$ that for every $j \geq j_{0}$ there is such $i(j) \geq n_{0}$ that for $n_{0} \leq i \leq i(j)$

$$
\mathbf{x}_{n_{0}}^{j} \in \operatorname{int}\left(I_{k}\right) \text { and } \mathbf{x}_{i}^{j} \in I_{k} \text { and } \mathbf{x}_{i(j)+1}^{j} \notin I_{k} .
$$

Let $\left\{\mathbf{x}_{i(j)}^{j}\right\}_{j=j_{0}}^{\infty}$ have a limit. Set $y=\lim _{j \rightarrow \infty} \mathbf{x}_{i(j)}^{j}$.

If $y \geq p$, then $y>p$ and $f(y)>y$ (if $y \leq p$ then $y<p$ and $f(y)<y$ ) and $\langle p ; f(y)\rangle^{*}$ is a nontrapping neighborhood of $p$.

Proof. Without loss of generality let $y \geq p$. Then $f(y) \notin \operatorname{int}\left(I_{k}\right)$ by continuity of $f$ and (7). Hence we have $y>p$ (because $p \in \operatorname{Fix}(f)$ by Lemma 3).

Suppose that $f(y)<y$. Then $f(y)<p$ and since $p$ is the limit point of $f^{n}(x)$ and $y$ is the limit point of $\mathbf{x}_{i(j)}^{j}$, then there is such $j_{1} \geq j_{0}$ that for all $j \geq j_{1}$ there is $s \in\left\{n_{0}, \ldots, i(j)\right\}$ such that

$$
\mathbf{x}_{i(j)+1}^{j}<\frac{p+f(y)}{2}<\mathbf{x}_{s}^{j}<\frac{p+y}{2}<\mathbf{x}_{i(j)}^{j} .
$$

Then in the same way as in Lemma 5 we can find a $z_{j} \in\left\langle d_{k-1} ; \mathbf{x}_{i(j)}^{j}\right\rangle$ such that $\mathbf{x}_{i(j)}^{j} \in f_{\delta}\left(z_{j}\right)($ see $(7))$. 
Hence by (8), for every $j \geq j_{1}$ we have

$$
\mathbf{x}_{i(j)+1}^{j} \leq z_{j} \leq \mathbf{x}_{i(j)}^{j} \quad \text { where }\left|\mathbf{x}_{i(j)}^{j}-\mathbf{x}_{i(j)+1}^{j}\right|>\frac{y-f(y)}{2}
$$

which is a contradiction to Lemma 4.

Hence we have $f(y) \geq y$ and since $d_{k} \notin \operatorname{Fix}(f)$ we have $f(y)>y$.

To prove the second part of the lemma we observe that $\langle p ; y\rangle$ is a nontrapping neighborhood of $p$.

Assume to the contrary that $\langle p ; y\rangle$ is not a nontrapping neighborhood of $p$.

Then there is a $z \in\langle p ; y\rangle$ such that $z \notin f(\langle p ; z\rangle)$ and we have for all $z_{1} \in f(\langle p ; z\rangle) ; z_{1}<z$. If we take $i$ sufficiently large $\left(\delta_{i}\right.$ sufficiently small), then $\mathbf{x}_{k}^{i} \in\langle p ; z\rangle$ implies $\mathbf{x}_{k+1}^{i} \leq z-\varepsilon_{z}$ where $\varepsilon_{z}>0$ and $z-\varepsilon_{z}>p$ (clearly $z \neq p)$.

Hence if $\mathbf{x}_{k+1}^{i}>z-\varepsilon_{z}$ (such an $\mathbf{x}_{k+1}^{i}$ exists because $y=\lim _{i \rightarrow \infty} \mathbf{x}_{i(j)}^{j}$ ) then $\mathbf{x}_{k}^{i}<p$ and by uniform continuity we can find $i_{0} \in N$ and $\omega>0$ such that $\mathbf{x}_{k+1}^{i}>z-\varepsilon_{z}$ implies $\mathbf{x}_{k}^{i}<p-\omega$ for $i \geq i_{0}$.

Then similarly as above we can find such $i_{1} \geq i_{0}$ that for all $i \geq i_{1}$ there are such $j, k \in N$ that

$$
\begin{gathered}
\mathbf{x}_{j+k}^{i}<p-\omega<\mathbf{x}_{j}^{i}<z-\varepsilon_{z}<\mathbf{x}_{j+k+1}^{i} \\
\mathbf{x}_{j+l}^{i}<z-\varepsilon_{z} \text { for } 0 \leq l \leq k
\end{gathered}
$$

and the same argument as above leads to contradiction to Lemma 4.

Hence $\langle p ; y\rangle$ is a nontrapping neighborhood of $p$ and clearly $\langle p ; f(y)\rangle$ has the same property.

Main Theorem. Let $f \in C^{0}(I ; I), \operatorname{Per}(f)=\operatorname{Fix}(f)$, and $\operatorname{Fix}(f)$ be nowhere dense. Then the following statements are equivalent.

(i) If for some $x \in I f^{n}(x)$ converges to the fixed point $p$ and there exists $a$ nontrapping neighborhood $\langle p ; q\rangle^{*}$ of $p$, then for all $O_{x}$ (neighborhoods of $x)$ and for all $z \in\langle p ; q)^{*}$ there is an $n \in N$ such that $\langle p ; z\rangle^{*} \subset$ $f^{n}\left(O_{x}\right)$.

(ii) $f$ has the shadowing property.

Proof of $\Downarrow$. Suppose that $f$ does not have the shadowing property. Then there exists an $\varepsilon>0$ and a sequence of $\delta$-chains $\left\{\mathbf{X}_{\delta_{j}}\right\}_{j=1}^{\infty}$ where $\delta_{j}<1 / j$ and $\mathbf{X}_{\delta_{j}}$ is not $\varepsilon$-shadowed.

Now we find the division of $I$ as in Lemma 5 with the property

$$
\max _{0 \leq i \leq n-1}\left|d_{i+1}-d_{i}\right|<\varepsilon .
$$

(Here we used the assumption that $\operatorname{Fix}(f)$ is nowhere dense.)

According to Lemma 6 there is such a $\delta>0$ and $N_{0} \in N$ that an interval index $K$ of any $\mathbf{X}_{\delta}$ is less than $N_{0}$. Without loss of generality we can assume that $\delta_{j} \leq \delta$ for all $j \in N$ and so

$$
K_{j}<N_{0} \text { where } K_{j} \text { is the interval index of } \mathbf{X}_{\delta_{j}} .
$$

Put

$$
x=\lim _{j \rightarrow \infty} \mathbf{x}_{0}^{j}
$$


(if necessary we can consider the proper subsequence), i.e., $x$ is the limit point of "starting points" of $\mathbf{X}_{\delta_{j}}$. From Lemma 3 we have

$$
\lim _{n \rightarrow \infty} f^{n}(x)=p \text { and } p \in \operatorname{Fix}(f) \text { and } p \in \operatorname{int}\left(I_{k}\right) \text { for some } k \text {. }
$$

Since $f^{n}(x)$ converges to $p$, there exists $n_{0}$ such that

$$
f^{n}(x) \in \operatorname{int}\left(I_{k}\right) \text { for all } n \geq n_{0}
$$

and by continuity there is $j_{0} \in N$ such that for all $j \geq j_{0}$

$$
\begin{gathered}
\left|\mathbf{x}_{i}^{j}-f^{i}(x)\right|<\varepsilon \text { for } 0 \leq i \leq n_{0} \\
\mathbf{x}_{n_{0}}^{j} \in \operatorname{int}\left(I_{k}\right)
\end{gathered}
$$

If there is such an $s \geq j_{0}$ that for all $i>n_{0} ; \mathbf{x}_{i}^{s} \in I_{k}$ then clearly $\mathbf{X}_{\delta_{s}}$ is $\varepsilon$-shadowed by orbit of $x$ which is in contradiction with our assumption. Hence for all $j \geq j_{0}$ there is $i(j) \geq n_{0}$ such that

$$
\mathbf{x}_{i(j)}^{j} \in I_{k} \quad \text { and } \quad \mathbf{x}_{i(j)+1}^{j} \notin I_{k}
$$

and let us consider only the smallest numbers with this property. Hence for $j \geq j_{0}$ we have

$$
\mathbf{x}_{n_{0}}^{j} \in \operatorname{int}\left(I_{k}\right) \text { and } \mathbf{x}_{i}^{j} \in I_{k} \text { and } \mathbf{x}_{i(j)+1}^{j} \notin I_{k} \text { for } n_{0} \leq i \leq i(j) .
$$

Set

$$
y=\lim _{j \rightarrow \infty} \mathbf{x}_{i(j)}^{i}
$$

(if necessary we can consider the proper subsequence).

Without loss of generality assume $y \geq p$. Then by (11) to (15) and by Lemma 8 we have $p<y<f(y)$ and $\langle p ; f(y)\rangle$ is the nontrapping neighborhood of $p$.

Now we can find $n_{1} \geq n_{0}$ such that $f^{n}(x) \leq p$ for all $n \geq n_{1}$ (it is possible by Lemma 7) and by continuity there are $j_{1} \geq j_{0}, \alpha>0$ such that for all $j \geq j_{1}$

$$
\begin{gathered}
\left|\mathbf{x}_{i}^{j}-f^{i}(z)\right|<\varepsilon \quad \text { for } 0 \leq i \leq n_{1}, \quad z \in\langle x-\alpha ; x+\alpha\rangle=O_{\alpha} \\
f^{n_{1}}\left(O_{\alpha}\right) \subset\left\langle d_{k-1} ; \frac{p+y}{2}\right\rangle .
\end{gathered}
$$

According to our assumption there exists an $n_{\alpha} \in N$ such that $\langle p$; $(y+f(y)) / 2\rangle \subset f^{n_{\alpha}}\left(O_{\alpha}\right)$ and so there is a neighborhood $O_{y}$ of $y$ such that

$$
O_{y} \subset f^{n_{\alpha}}\left(O_{\alpha}\right) \text { and } O_{y} \subset\left\langle\frac{p+y}{2} ; d_{k}\right\rangle \text {. }
$$

Since $\langle p ; f(y)\rangle$ is a nontrapping neighborhood of $p$, we obtain that $O_{y} \subset$ $f^{n_{\alpha}+l}\left(O_{\alpha}\right)$ for all $l \geq 0$.

Claim. We claim that for every number $n_{\alpha}+l$ there is a neighborhood $O_{\beta}=$ $\left\langle x-\beta_{1} ; x+\beta_{2}\right\rangle ; O_{\beta} \subset O_{\alpha}$, such that

(*) $f^{s}\left(O_{\beta}\right) \subset I_{k}$ for $n_{1} \leq s \leq n_{\alpha}+l$

(**) $O_{y} \subset f^{n_{\alpha}+l}\left(O_{\beta}\right)$. 
Proof. We have $f^{n_{1}}\left(O_{\alpha}\right) \subset I_{k}$ and by the continuity of $f$ we can find such an $O_{\alpha_{1}}=\left\langle x-\alpha_{1} ; x+\alpha_{2}\right\rangle \subset O_{\alpha}$ that $f^{n_{1}+1}\left(O_{\alpha_{1}}\right) \subset I_{k}$ and $O_{\alpha_{1}}$ is maximal with these properties (we simply "cut off" $f^{n_{1}+1}\left(O_{\alpha}\right) \backslash I_{k}$ ) and by induction we can find $O_{\alpha_{1}} \subset O_{\alpha_{i-1}}$ with $f^{n_{1}+i}\left(O_{\alpha_{i}}\right) \subset I_{k}$ and finally for $i=n_{\alpha}+l-n_{1}$ we obtain $O_{\beta}$ with the property $(*)$.

If $f^{n_{\alpha}+l}\left(O_{\alpha} \backslash O_{\beta}\right) \cap O_{y}=\varnothing$ then we have $O_{y} \subset f^{n_{\alpha}+l}\left(O_{\beta}\right)$ (see (18)).

Suppose now that there is a $z \in f^{n_{\alpha}+l}\left(O_{\alpha} \backslash O_{\beta}\right) \cap O_{y}$. Then there exists such a $z_{1} \in O_{\alpha} \quad\left(z_{1} \in f^{-\left(n_{\alpha}+l\right)}(z)\right)$ that

$$
f^{n_{1}}\left(z_{1}\right) \in\left\langle d_{k-1} ; \frac{p+y}{2}\right\rangle \text { and } f^{n_{\alpha}+l}\left(z_{1}\right) \in\left\langle\frac{p+y}{2} ; d_{k}\right\rangle \text { and } f^{s}\left(z_{1}\right) \notin I_{k}
$$

for some $n_{1}<s<n_{\alpha}+l$ (see (17), (18)). Let $s$ be the smallest number with this property. From Lemma 2 we obtain that $f^{s}\left(z_{1}\right)>d_{k}$. Because $f^{s}(x) \leq p$ then $\left\langle p ; d_{k}\right\rangle \subset f^{s}\left(O_{\alpha_{s-n_{1}}}\right) \quad(s$ was chosen to be the smallest one) and since $\left\langle p ; d_{k}\right\rangle$ is a nontrapping neighborhood of $p$ (because $\left.\left\langle p ; d_{k}\right\rangle \subset\langle p ; f(y)\rangle\right)$ we obtain that $O_{y} \subset f^{n_{\alpha}+l}\left(O_{\beta}\right)$.

Take such an $r_{1} \geq j_{1}$ that for all $j \geq r_{1} ; i(j)>n_{\alpha}$. Then for any $\mathbf{X}_{\delta_{j}}$, $j \geq r_{1}$; according to (9), (14), (16) and the claim we can find an interval $R_{j}^{1} \subset O_{\alpha}$ such that for all $z \in R_{j}^{1}$

$$
O_{y} \subset f^{i(j)}\left(R_{j}^{1}\right) \text { and }\left|f^{s}(z)-\mathbf{x}_{s}^{j}\right|<\varepsilon \quad \text { for } 0 \leq s \leq i(j) .
$$

Now we have the sequence of $\delta$-chains $\left\{\mathbf{X}_{\delta_{j}}\right\}_{j \geq r_{1}}^{\infty}$ with starting points $\mathbf{x}_{i(j)}^{j}$ and $\lim _{j \rightarrow \infty} \mathbf{x}_{i(j)}^{j}=y$. Again these $\delta_{j}$-chains $\left\{\mathbf{x}_{i}^{j}\right\}_{i=i(j)}^{\infty}$ are not $\varepsilon$-shadowed by any points of $O_{y}$ for all $j \geq r_{1}$. Indeed, if there is such a $j \geq r_{1}$ that $\left\{\mathbf{x}_{i}^{j}\right\}_{i=i(j)}^{\infty}$ is $\varepsilon$-shadowed by some point of $O_{y}$, then from (19) we have that this $\mathbf{X}_{\delta_{j}}$ is $\varepsilon$-shadowed by some point of $R_{j}^{1}$ which is in contradiction with our assumption.

So we have the same situation as in the beginning, but the very important property is that $\mathbf{x}_{s}^{j} \in \operatorname{int}\left(I_{k}\right)$ for some $s \in\{0, \ldots, i(j)\}$ and $\mathbf{x}_{i(j)+1}^{j} \notin I_{k}$ for all $j \geq r_{1}$. So we have that $K_{j} \geq 1$ for all $j \geq r_{1}$.

If we repeat this procedure once more, we obtain that there are $r_{2} \geq r_{1}$, $y_{2} \in I_{k_{2}}$, and $O_{y_{2}}$ a neighborhood of $y_{2}$ such that for all $j \geq r_{2}$ there are such $i(j)_{2} \geq i(j)$ and $R_{j}^{2} \subset O_{y}$ that for all $z \in R_{j}^{2}$

$$
\begin{gathered}
O_{y_{2}} \subset f^{i(j)_{2}}\left(R_{j}^{2}\right) \\
\left|f^{s}(z)-\mathbf{x}_{s}^{j}\right|<\varepsilon \text { for } i(j) \leq s \leq i(j)_{2} \\
\lim _{j \rightarrow \infty} \mathbf{x}_{i(j)_{2}}^{j}=y_{2}
\end{gathered}
$$

$\left\{\mathbf{x}_{i}^{j}\right\}_{i=i(j)_{2}}^{\infty}$ is not $\varepsilon$-shadowed by any points of $O_{y}$

and, of course, we have

$$
K_{j} \geq 2 \text { for all } j \geq r_{2} \text {. }
$$

But if we repeat this procedure $N_{0}$ times we obtain that there is such an $r_{N_{0}} \in N$ that $K_{j} \geq N_{0}$ for all $j \geq r_{N_{0}}$ which is a contradiction to (10). 
Proof of $\Uparrow$. Let $x \in I, f^{n}(x) \rightarrow p$, and $\langle p ; q\rangle^{*}$ be a nontrapping neighborhood of $p$ and let there exist a $z \in\langle p ; q\rangle^{*}$ and a neighborhood $O_{x}$ of $x$ such that

$$
\langle p ; z\rangle^{*} \not \subset f^{n}\left(O_{x}\right) \text { for all } n \in\{0,1, \ldots\} .
$$

Without loss of generality assume that $\langle p ; q\rangle^{*}=\langle p ; q\rangle$. Let $\varepsilon>0$ such that

$$
|q-z|>\varepsilon \text { and }\langle x-\varepsilon ; x+\varepsilon\rangle \subset O_{x} .
$$

Now we claim that for any $\delta>0$ there exists $\mathbf{X}_{\delta}$ which is not $\varepsilon$-shadowed.

By Lemma 7 there is an $n_{\delta} \in N$ such that

$$
\begin{gathered}
f^{n}(x) \leq p \quad \text { for all } n \geq n_{\delta} \\
\left|p-f^{n_{\delta}}(x)\right|<\delta .
\end{gathered}
$$

Let $k_{\delta} \in N$ be such that $k_{\delta}>|q-p| / \delta$. Set $\mathbf{x}_{n_{\delta}+k_{\delta}}=q$ and for $i \in$ $\left\{1, \ldots, k_{\delta}\right\}$ we define:

$$
\begin{aligned}
& \text { if } \mathbf{x}_{n_{\delta}+k_{\delta}-i+1}-\delta \leq p \text { then } \mathbf{x}_{n_{\delta}+k_{\delta}-i}=p \\
& \text { if } \mathbf{x}_{n_{\delta}+k_{\delta}-i+1}-\delta>p \text { then } f\left(\mathbf{x}_{n_{\delta}+k_{\delta}-i}\right)=\mathbf{x}_{n_{\delta}+k_{\delta}-i+1}-\delta \text { and } \mathbf{x}_{n_{\delta}+k_{\delta}-i} \in \\
& \left\langle p ; \mathbf{x}_{n_{\delta}+k_{\delta}-i+1}-\delta\right\rangle
\end{aligned}
$$

(this definition is correct because $\langle p ; q\rangle$ is a nontrapping neighborhood of $p$ ) and let $\mathbf{x}_{i}=f^{i}(x)$ for $i \in\left\{0, \ldots, n_{\delta}-1\right\}$.

Hence $\left\{\mathbf{x}_{i}\right\}_{i=0}^{n_{\delta}+k_{\delta}}$ is a $\delta$-chain (see (23)). If $y \notin O_{x}$ then $\left|y-\mathbf{x}_{0}\right|>\varepsilon$ (see (21)) and if $y \in O_{x}$ then $f^{n_{\delta}+k_{\delta}}(y)<z$ (see (20) and (22)) and so $\mid \mathbf{x}_{n_{\delta}+k_{\delta}}$ $f^{n_{\delta}+k_{\delta}}(y) \mid>\varepsilon($ see $(21))$.

So our $\delta$-chain cannot be $\varepsilon$-shadowed.

Remark 1. From the Main Theorem we obtain that if $f$ has the shadowing property, $\operatorname{Per}(f)=\operatorname{Fix}(f)$ and $p \in \operatorname{Fix}(f)$, then the following condition is fulfilled:

If $\langle p ; q\rangle^{*}$ is the maximal nontrapping neighborhood of $p$, then there exists such a sequence $\left\{x_{i}\right\}_{i=-\infty}^{\infty}$ that $f\left(x_{i-1}\right)=x_{i}, \lim _{i \rightarrow-\infty} x_{i}=p$, and

$$
\inf \left\{\left|x_{i}-q\right| ; i \in Z\right\}=0 \text {. }
$$

If we consider only nondecreasing maps then this condition is equivalent to the shadowing property and thus with the result of [3]. This means that there cannot be such a fixed point $p$ with a neighborhood $O_{p}$ that for all $x \in O_{p}$; $f(x) \geq x$ (or for all $\left.x \in O_{p} ; f(x) \leq x\right)$ (see Figure 1).

Lemma 9. Let $f \in C^{0}(I, I)$ and $n \in N$. Then $f$ has the shadowing property if and only if $f^{n}$ has the shadowing property.

Proof.

$\Rightarrow$ If $\left\{\mathbf{x}_{i}^{n}\right\}_{i=0}^{\infty}$ is a $\delta$-chain of $f^{n}$ then $\left\{\mathbf{x}_{i}^{n}, f\left(\mathbf{x}_{i}^{n}\right), \ldots, f^{n-1}\left(\mathbf{x}_{i}^{n}\right)\right\}_{i=0}^{\infty}$ is a $\delta$-chain of $f$ and we are done.

$\Leftarrow$ From uniform continuity we obtain that

(*) for all $\varepsilon>0$ there is such $\delta, \varepsilon_{n}>0$ that if $\left\{\mathbf{x}_{i}\right\}_{i=0}^{\infty}$ is $\delta$-chain and $\left\{\mathbf{x}_{n i}\right\}_{i=0}^{\infty}$ is $\varepsilon_{n}$-shadowed by $f^{n}$, then $\left\{\mathbf{x}_{i}\right\}_{i=0}^{\infty}$ is $\varepsilon$-shadowed by $f$; 


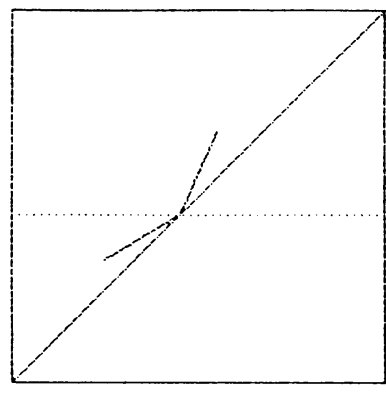

FIGURE 1

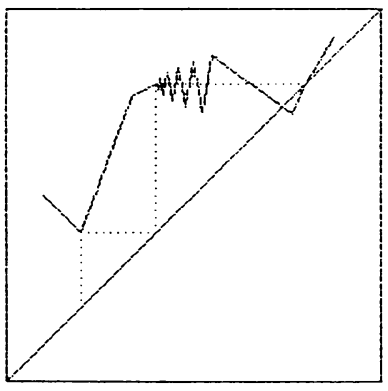

FIGURE 2

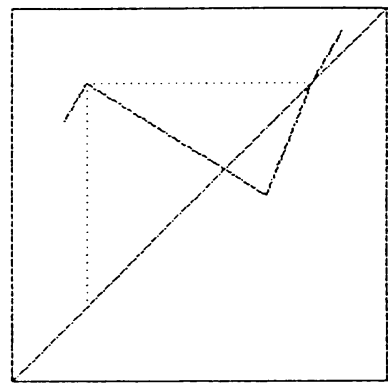

FigURE 3

(**) for all $\delta_{n}>0$ there is such $\delta \in\left(0 ; \delta_{n}\right\rangle$ that if $\left\{\mathbf{x}_{i}\right\}_{i=0}^{\infty}$ is $\delta$-chain of $f$ than $\left\{\mathbf{x}_{n i}\right\}_{i=0}^{\infty}$ is $\delta_{n}$-chain of $f^{n}$.

From here we are easily done.

Definition 3. We will call a one-side neighborhood $\langle p ; q\rangle^{*}$ of the periodic point $p$ a $m$-nontrapping neighborhood of $p$ if $f^{m}(p)=p$ and for every $x \in$ $\langle p ; q\rangle^{*}, x \in f^{m}\left(\langle p ; x\rangle^{*}\right)$.

Theorem 2. Let $f \in C^{0}(I ; I)$ and $\operatorname{Per}(f)$ be a closed (i.e., $f$ is of type $\left.2^{n}\right)$ nowhere dense set. Then the following statements are equivalent:

(i) If for some $x \in I f^{n}(x)$ converges to the periodic point $p$ and there exists an m-nontrapping neighborhood $\langle p ; q\rangle^{*}$ of $p$, then for all $O_{x}$ (neighborhoods of $x$ ) and for all $z \in\langle p ; q\rangle^{*}$ there is an $n \in N$ such that $\langle p ; z\rangle^{*} \subset f^{m n}\left(O_{x}\right)$.

(ii) $f$ has the shadowing property.

Proof. The proof is the straightforward consequence of the Main Theorem and Lemma 9 (if $f$ is a map of type $2^{n}$ then $f^{2^{n}}$ is a map with $\operatorname{Per}\left(f^{2^{n}}\right)=$ $\left.\operatorname{Fix}\left(f^{2^{n}}\right)\right)$.

Remark 2. The condition (i) is not fulfilled, in particular, when some critical point is eventually mapped in a "wrong way" (for "good way" see Figure 2) onto the repelling periodic point (see Figure 3).

However, this condition needn't be satisfied by a lot of maps from some family of maps. For instance, according to Lemma 7.3 in [1], for the family of tent maps the set of those parameters, for which the critical point is mapped onto a periodic point (which is in fact repelling), is dense in the set of all considered parameters.

In this section we prove that chaotic maps of the type $2^{\infty}$ do not have the shadowing property. We begin with

Definition 4. Let $f \in C^{0}(I, I)$. Then $u, v \in I$ are $f$-separable points if there are disjoint $f$-periodic intervals $J_{u}, J_{v} \subset I$ with $u \in J_{u}, v \in J_{v}$. Otherwise $u, v$ are $f$-nonseparable. 
Lemma 10. (Janková and Smítal [2]) Let $f \in C^{0}(I, I)$. Then $f$ is chaotic if and only if there is an infinite attractor containing two $f$-nonseparable points.

Lemma 11. (Smítal [5]) Let $f \in C^{0}(I, I)$ be of type $2^{\infty}$ and let $\omega$ be an infinite attractor of $f$. Then there is a sequence $\left\{J_{k}\right\}_{k=0}^{\infty}$ of periodic intervals with the following properties:

(i) $J_{k}$ has period $2^{k}$

(ii) $J_{k+1} \subset J_{k}$

(iii) $\omega \subset \bigcup_{i=1}^{2^{k}} f^{i}\left(J_{k}\right)$.

Theorem 3. Let $f \in C^{0}(I, I)$ be a chaotic map of the type $2^{\infty}$. Then $f$ does not have the shadowing property.

Proof. By Lemma 10 there is an infinite attractor $\omega$ containing two $f$-nonseparable points $p, q \in \omega$. By Lemma 11 there is a sequence $\left\{J_{k}\right\}_{k=0}^{\infty}$ which has the properties (i)-(iii). Without loss of generality let $p \in J_{k}$ for all $k$. If there is such $k \in N$ that $q \notin J_{k}$ then $p, q$ are $f$-separable which is a contradiction. Hence

$$
p, q \in J_{k} \quad \text { for } k=0,1, \ldots
$$

Let $J=\bigcap_{k=0}^{\infty} J_{k}$. From Lemma 11 we obtain that

$$
J \cap f^{i}(J)=\varnothing \quad \text { for } i \in N .
$$

Set $\varepsilon=|p-q| / 2, \mathbf{x}_{0}=(p+q) / 2$, and let $\delta>0$. Then by Lemma 11 there are $j, k \in N$ such that

$$
\left|f^{j}\left(J_{k}\right)\right|<\delta .
$$

But there is $\mathbf{x}_{j} \in f^{j}\left(J_{k}\right)$ such that $f^{2^{k}-j}\left(\mathbf{x}_{j}\right)=\mathbf{x}_{0}$ (see (24)). Set

$$
\begin{aligned}
\mathbf{x}_{i}=f^{i}\left(\mathbf{x}_{0}\right) & \text { for } 0 \leq i \leq j-1 \\
\mathbf{x}_{j+i}=f^{i}\left(\mathbf{x}_{j}\right) & \text { for } 0 \leq i \leq 2^{k}-j .
\end{aligned}
$$

We have that $\left\{\mathbf{x}_{i}\right\}_{i=0}^{2^{k}}$ is a $\delta$-chain (see (26)).

If $y \notin J$ then $\left|y-\mathbf{x}_{0}\right|>\varepsilon$ and if $y \in J$ then $\left|f^{2^{k}}(y)-\mathbf{x}_{2^{k}}\right|>\varepsilon$ (see (25)). Hence we find a $\delta$-chain which is not $\varepsilon$-shadowed.

\section{REFERENCES}

1. E. M. Coven, I. Kan, and J. A. Yorke, Pseudo-orbit shadowing in the family of tent maps, Trans. Amer. Math. Soc. 308 (1988), 227-241.

2. K. Janková and J. Smital, Characterization of chaos, Bull. Austral. Math. Soc. 34 (1986), 283-292.

3. T. Pennings and J. Van Eeuwen, Pseudo-orbit shadowing on the unit interval, preprint, 1990.

4. A. N. Šarkovskiī, On cycles and the structure of a continuous mapping, Ukrain. Mat. Z. 17 (1965), 104-111. (Russian)

5. J. Smital, Chaotic functions with zero topological entropy, Trans. Amer. Math. Soc. 297 (1986), 269-282. 
Department of Mathematics, SvF SVŠt, Radlinského 11, 81368 Bratislava, Czechoslovakia

and

Department of Mathematics, Georgia Institute of Technology, Atlanta, Georgia 30332

Mathematical Institute, Slovak Academy of Sciences, Štefánikova 49, 81473 Bratislava, Czechoslovakia 